\title{
A New Image Decomposition and Reconstruction Approach -- Adaptive Fourier Decomposition
}

\author{
Can He, Liming Zhang \\ Faculty of Science and Technology, University of Macau, \\ Avenida da Universidade, Taipa, Macau, China \\ Xiangjian $\mathrm{He}$ \\ Faculty of Engineering \& Information Technology \\ University of Technology, Sydney, Australia
}

\begin{abstract}
Fourier has been a powerful mathematical tool for representing a signal into an expression consist of sin and cos. Recently a new developed signal decomposition theory is proposed by Pro. Tao Qian named Adaptive Fourier Decomposition (AFD), which has the advantage in time frequency over Fourier decomposition and without the need for a fixed window size problem such as short-time frequency transform (STFT). Studies show that AFD can fast decompose signals into positive-frequency functions with good analytical properties. In this paper we apply AFD into image decomposition and reconstruction area first time in the literature, which shows a promising result and gives the fundamental prospect for image compression.
\end{abstract}

Keywords: Adaptive Fourier decomposition, signal processing, image compression, image decomposition, mono-components

\section{Introduction}

Fourier transform expands a signal into infinite series, after it stops through some given threshold, we can get the reconstructed signal by the inverse transform [13]. Adaptive Fourier Decomposition (AFD) is a newly proposed signal processing theory that has significant influence in signal denoising and control theory[4]. 'Mono-components' is brought up here to represent signals decomposed at each level. There are two ways that can decompose signal into mono-components with positive frequencies [5], which makes it possible to have some applications that are related to some mathematical analysis of signals. Maximal Selection Principle (MSP) is used here to get the mono-components [6,7]. AFD first generates a large pool of mono-components, then it decomposes the signal by using these components in the pool. Fourier decomposition is a special case of AFD, when all the corresponding parameters in AFD are all chosen to be zero; it will be in the form of Fourier transform. By doing the inverse transform, AFD can also get the approximate signal [8]. 
AFD keeps most good characteristics of Fourier transform. In general, AFD can be applied in all applications in which Fourier transform can be used. The advantage of AFD is that the decomposed components have distinct division for different frequencies, which can be further used for distinguishing different frequency components of signals. Signals decomposed by Fourier Transform (FT) in the sum of trigonometric functions do not have such good time-varying timefrequency instantaneous frequency (IF).

AFD can provide many potential applications in image processing. With the better and faster converging properties, AFD can serve for some applications such as speech recognition, image denoising $[9,10]$, edge detection, image compression and so on. For a given signal, by summing up it's Fourier Series components, we can get the approximate signal. The more components participate in accumulation, the more likely they are. Noises are usually in the form of high frequencies. They come later no matter in Fourier expansion or AFD. Adding up some components decomposed in the preceding levels will take away the high frequency part. In another word, noise elimination can be done. Edge detection continues in this way, certain components which have some particular frequency from the AFD are selected, by add them together, we can get the desired signal -- edges.

Transformations are usually used in image compression. Discrete Cosine Transform (DCT) is used in the famous JPEG compression before quantization. Same as Fast Fourier Transformation (FFT), DCT is the transform from time domain to frequency domain. The difference is that there exists no complex number in the result. DCT first partitions the original image into $8 \times 8$ blocks. Every $8 \times 8$ blocks turns to be another $8 \times 8$ group through basis function. DCT concentrates most of the energy (low frequency) in the upper left corner of an image and lower right corner with less energy (high frequency). JPEG is a lossy compression that it deducts the high frequencies that are insensitive to our human eyes. Removing $50 \%$ of the high frequencies may only loss $5 \%$ of the encoding information. So if there are other transformations converging faster than the one currently used in JPEG, there would be less redundancy generated. AFD is one of the new transformations, which converges faster than Fourier transform does.

This paper introduces AFD into the image processing area first time in the literature. The proposed algorithm is compared with traditional Fourier transform The experiment results show that AFD converges much faster than Fourier transform. It provides potential capacity for further applications.

This paper is organized as follow. The principle of the AFD based image decomposition and reconstruction is introduced in Section 2. The experiment results are shown in Section 3. Conclusions are drawn in Section 4. 


\section{Principles of AFD based image decomposition and reconstruction approach}

\subsection{Brief overview of AFD}

AFD is based on the rational orthogonal system,or the the Takenaka-Malmquist system[4], where variables in Hardy Space $H^{2}(D)$ turn into linear combinations using (1) and modified blashchke products.

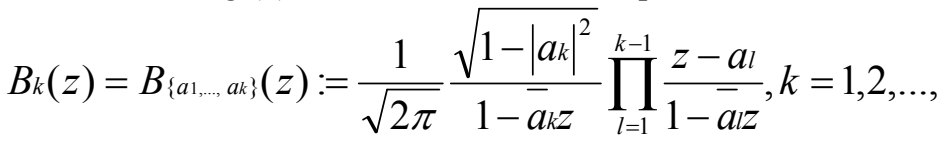

Here $B_{k}$ is what we called Takenaka-Malmquist (TM)system, $a_{k}$ is in the unit disk, $a_{k}$ is chosen by the decomposed signal[5,6]. Maximal Selection Principle(MSP) is used here to select $a_{1}$ first, then using the recursive formula to find $a_{2}, a_{3} \ldots$, , until we reach a certain threshold. $B_{k}$ is the so-called monocomponents or pre-mono-components, after multiplying $e^{i M t}$ where $M$ stands as positive number, it becomes mono-components. Here when all $a_{k}$ are chosen to be zero, then $B_{k}$ is the half of the Fourier system[7].

For general parameters $\left\{B_{k}\right\}$ is the basis for all $H^{P}(1 \leq P \leq \infty)$ satisfies (2),

$$
\sum_{k=1}^{\infty}\left(1-\left|a_{k}\right|\right)=\infty
$$

A TM system consisted by rational parameters in Hardy space can greatly estimate functions in the same Hardy space. $H$ is the Hilbert transform on the unit disk.

$$
f=2 \operatorname{Re} f^{+}-C_{0}
$$

Which $f=f^{+}+f^{-}$is the Hardy decomposition of $f \in L^{2}(\partial D)$,

$$
f^{+}=\frac{1}{2}(f+i H f)
$$

AFD will stop either the the algorithm has reached to the desired level or the energy difference has come to the accuracy $\varepsilon$.

$$
\left\|f^{+}\right\|-\sum_{k=1}^{N}\left|\left\langle f, B_{k}\right\rangle\right|^{2}<\varepsilon
$$

In our case, we set $a_{1}$ to be zero and the nth parameters is calculated through the previous arguments. 


\subsection{Decomposition comparison between AFD and FT}

In Fourier transform which decomposes any signals into the same basic trigonometric functions, the entrance $e^{i k t}$ for the Fourier expansion of a given signal may arrive late, which is an important part of the total energy. So the convergence is not so ideal [4].

In AFD, greedy algorithm has been applied. AFD decomposes the given signal into different mono-components [5,6]. The mono-components decomposed are selected based on the given signal by using Maximal Selection Principle (MSP). MSP means that for a given signal, the AFD algorithm starts with selecting a mono-component that is most close to the original signal in energy sense, which starts from the low frequency to high frequency. Then at each continuous selection, it applies the same energy principle to find each mono-component that draws near the remainder. It is the reason that the decomposition is said to be adaptive. The decomposition usually leads to fast convergence than what Fourier decomposition does[7].

\subsection{The algorithm of the proposed approach}

The flowchart of the proposed algorithm is illustrated in Fig. 2.

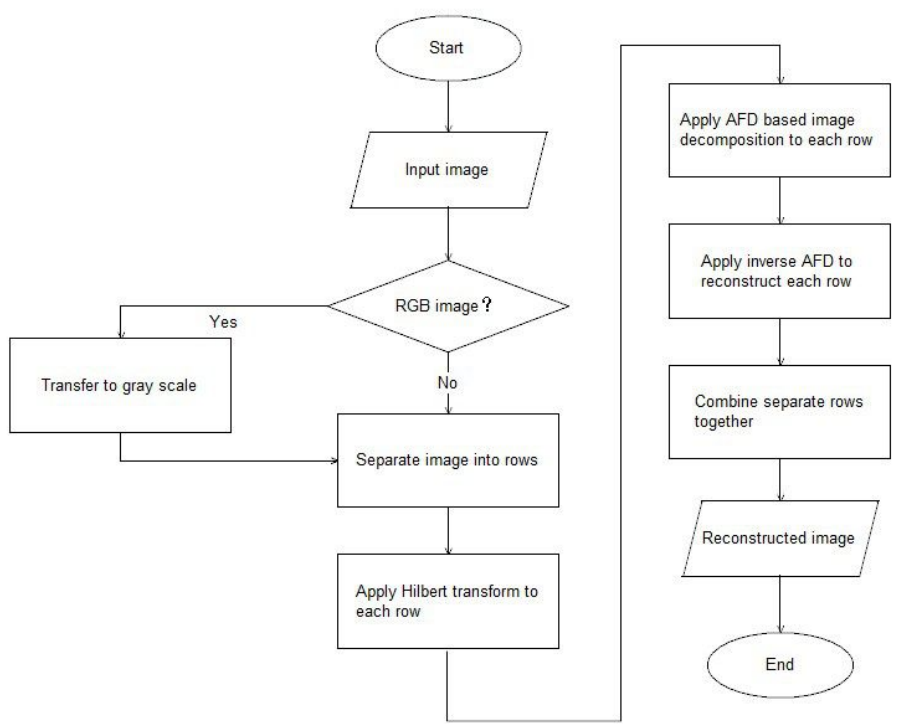

Fig. 2: The flowchart our method 
We can regard an image as different portions of signals according to its rows. So we disassemble the original image into different row and then apply AFD row by row. First we apply Hilbert transform to the rows to make the primitive function in a complex plane, and then handle it with AFD. The ultimate step is the combination of different rows into a whole image. The detailed algorithm is shown below. AFD code is released in Pro. Qian's Homepage http://www.fst.umac.mo/en/staff/documents/fsttq/afd_form/Index.html.

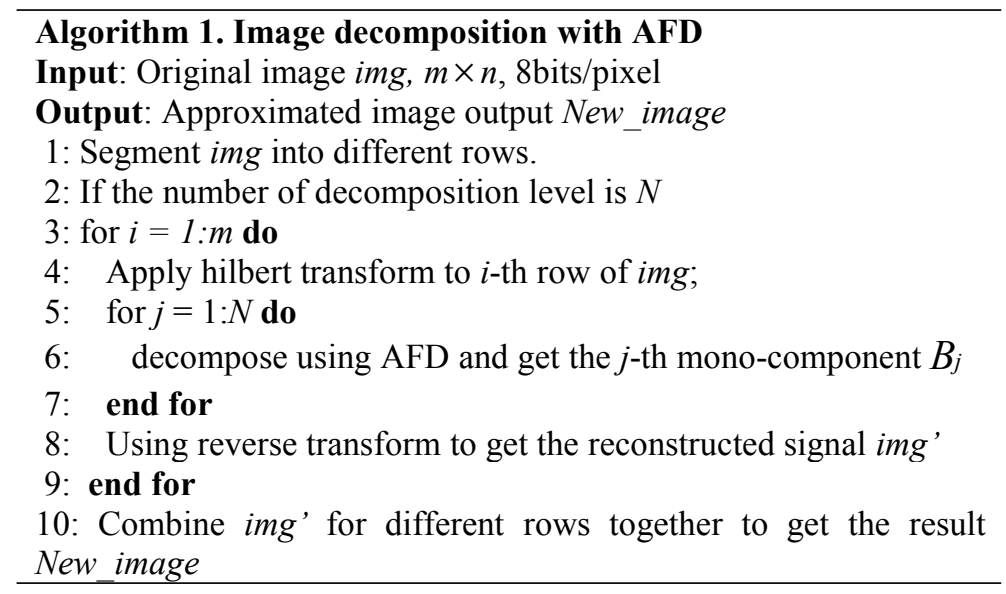

\section{Experimental results}

Usually there are three factors to be used to judge the quality of an image. They are Mean Square Error(MSE), Peak Signal to Noise Rate( PSNR) and ContourVolume (CV)[11].

I.The traditional objective rating of an image are MSE and PSNR. MSE first calculates the mean square of original picture and reconstructed picture, then evaluate the reconstructed image according to the result of the results. $\mathrm{M}$ and $\mathrm{N}$ is the width and height of the original image, $f_{i j}$ is the original image and $f_{i j}^{\prime}$ is the reconstructed image.

$$
M S E=\frac{1}{M \times N} \sum_{0 \leq i<N} \sum_{0 \leq j<M}\left(f_{i j}-f_{i j}{ }^{\prime}\right)^{2}
$$

II.PSNR is the most vital indicator for the evaluation of images based on communication theory. PSNR is the ratio of the maximum information and noise intensity. Here we assign L with 255 for dealing with discrete pixels.

$$
P S N R=10 \times \lg \frac{L \times L}{M S E}
$$

III.The more abundant the detail is , the more clear the images are, so is the 
edges. $\mathrm{CV}$ can tell the whether the image is clear or fuzzy, the bigger number indicates the good quality. First we use a $3 \times 3$ Laplacian window to extract the edges. Then count the pixels of edges, sum up the absolute value of the pixels and see the results.

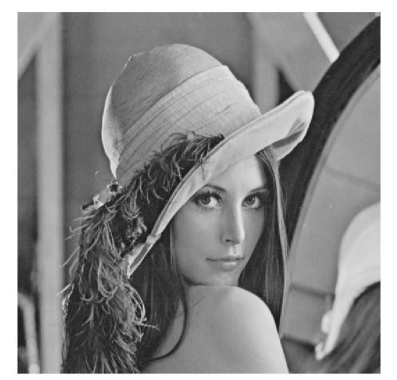

Fig. 3: Lena image

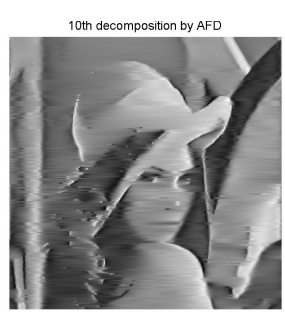

(a)

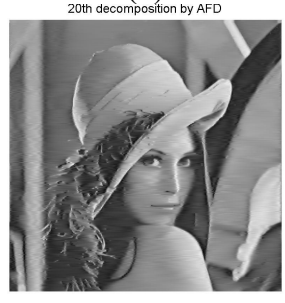

(c)

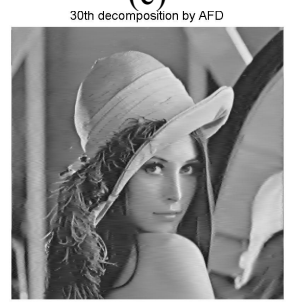

(e)

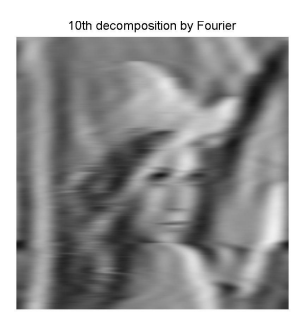

(b)

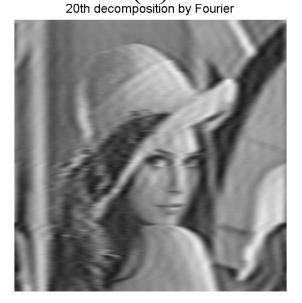

(d)

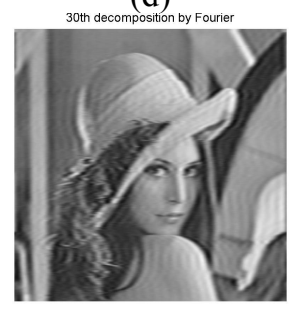

(f) 


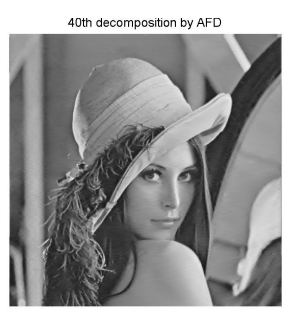

(g)

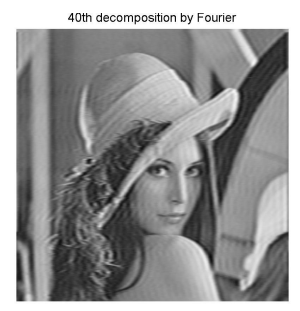

(h)

Fig. 4: Image decomposition by AFD (a)(c)(e)(g) and Fourier(b)(d)(f)(h)

Four experiments are conducted in this paper. The results are illustrated in Fig. 4, Table 1 and Table 2. The classical 'Lena' (see Fig. 3) with size $512 \times 512$, $8 \times 8$ bits/pixel is used. In Fig. 4 , the left side column is the reconstruction result by AFD, with $10^{\text {th }}, 20^{\text {th }}, 30^{\text {th }}$ and $40^{\text {th }}$ decomposition level. The corresponding results by using Fourier decomposition are shown in the right side column. We can see that at the same level, AFD based result appears more clearly than Fourier does. Through the objective outcomes in Table 1 and 2, for AFD, the MSE is lower than Fourier, the PSNR and CV is higher than Fourier. The overall experiment results indicate that AFD converges faster in image decomposition, which provides potential applications for image compression in the future.

Table 1: Comparing parameters of AFD

\begin{tabular}{|c|c|c|c|}
\hline AFD & MSE & PSNR & CV \\
\hline $10^{\text {th }}$ decomposition & 0.0036 & 24.4793 & 0.1518 \\
\hline $20^{\text {th }}$ decomposition & 0.0013 & 28.7611 & 0.1641 \\
\hline $30^{\text {th }}$ decomposition & $7.2924 \mathrm{e}-04$ & 31.3634 & 0.1707 \\
\hline $40^{\text {th }}$ decomposition & $4.7559 \mathrm{e}-04$ & 33.2158 & 0.1754 \\
\hline
\end{tabular}

Table 1: Comparing parameters of Fourier

\begin{tabular}{|c|c|c|c|}
\hline Fourier & MSE & PSNR & CV \\
\hline $10^{\text {th }}$ decomposition & 0.0087 & 20.5921 & 0.0773 \\
\hline $20^{\text {th }}$ decomposition & 0.0042 & 23.7185 & 0.1133 \\
\hline $30^{\text {th }}$ decomposition & 0.0027 & 25.7526 & 0.1345 \\
\hline $40^{\text {th }}$ decomposition & 0.0019 & 27.2116 & 0.1514 \\
\hline
\end{tabular}

\section{Conclusions}

This paper presents the principle of the AFD based image decomposition and reconstruction approach. Our experiment results show that AFD converges faster than Fourier transform does. Discrete cosine transform (DCT) is the real part of the Fourier transform, which is the foundation of JPEG compression. Since DCT 
has proved its advantages in JPEG, AFD can also find its corresponding transform to replace DCT, which can be another promising method for lossy image compression.

\section{Corresponding Author}

Can He, rahxphoon@gmail.com, Master Student, University of Macau Liming Zhang, lmzhang@umac.mo, Assistant Professor, University of Macau Xiangjian He,xiangjian.he@uts.edu.au, Full Professor, University of Technology, Sydney

\section{References}

[1] Fienup, James R. "Reconstruction of an object from the modulus of its Fourier transform." Optics letters 3.1 (1978): 27-29.

[2] Shepp, Lawrence A., and Benjamin F. Logan. "The Fourier reconstruction of a head section." Nuclear Science, IEEE Transactions on 21.3 (1974): 21-43.

[3] Tsai, Du-Ming, and Wei-Yao Chiu. "Motion detection using Fourier image reconstruction." Pattern Recognition Letters 29.16 (2008): 2145-2155.

[4] Qian, Tao, Liming Zhang, and Zhixiong Li. "Algorithm of adaptive Fourier decomposition." Signal Processing, IEEE Transactions on 59.12 (2011): 5899-5906.

[5] Qian, Tao, Yan-Bo Wang, and Pei Dang. "Adaptive decomposition into mono-components." Advances in Adaptive Data Analysis 1.04 (2009): 703 709.

[6] Qian, Tao. "Mono-components for decomposition of signals." Mathematical methods in the applied sciences 29.10 (2006): 1187-1198.

[7] Qian, Tao, Hong Li, and Michael Stessin. "Comparison of adaptive monocomponent decompositions." Nonlinear Analysis: Real World Applications 14.2 (2013): 1055-1074.

[8] Qian, Tao, and Yan-Bo Wang. "Adaptive Fourier series - a variation of greedy algorithm." Advances in Computational Mathematics 34.3 (2011): 279-293.

[9] Adaptive Fourier Decomposition Based Signal Instantaneous Frequency Computation Approach

[10] Zhang, Liming. "A New Time-Frequency Speech Analysis Approach Based On Adaptive Fourier Decomposition." Proceedings of XXXIV. International Conference on Information and Computer Sciences, Zurich, Swissland 30-31 July, 2013.

[11] Wang, Zhou, and Alan C. Bovik. "A universal image quality index." Signal Processing Letters, IEEE 9.3 (2002): 81-84. 